\title{
A satisfação de pacientes em tratamento dialítico com relação aos cuidados do enfermeiro
}

\author{
Dialysis patient satisfaction with nursing care \\ La satisfacción de pacientes en diálisis en relación con los cuidados del enfermero
}

\author{
Ingrid Fernanda de Oliveira Vieiral; Felipe Kaezer dos Santos"'; Frances Valéria Costa e SilvallI; \\ Silvia Maria de Sá Basilio Lins'vi ; Natasha Cristina Cunha Muniz ${ }^{V}$
}

\begin{abstract}
RESUMO
Objetivo: analisar a satisfação dos pacientes em tratamento dialítico com relação aos cuidados de enfermagem. Método: estudo descritivo, de abordagem quantitativa de campo, realizado de maio a agosto de 2016, em um hospital universitário do Rio de Janeiro. A amostra foi de 37 pacientes que realizam hemodiálise ou diálise peritoneal no hospital por tempo igual ou superior a três meses, maiores de 18 anos. Utilizou para a coleta de dados o Instrumento de Satisfação do Paciente. Resultados: a média de satisfação geral dos pacientes foi de 3,89 para os três itens: educação, confiança e teórico-prático, sendo que os pacientes da hemodiálise apresentaram a média de satisfação de 3,69 e os pacientes da diálise peritoneal de 4,44. Conclusão: os pacientes em tratamento dialítico do hospital se encontram satisfeitos com os cuidados de enfermagem que lhes são prestados.

Descritores: Satisfação do paciente; enfermagem; nefrologia; cuidados de enfermagem
\end{abstract}

\section{ABSTRACT}

Objective: to examine dialysis patient satisfaction with related nursing care. Method: this descriptive study was conducted, on a quantitative field approach, from May to August 2016, at a university hospital in Rio de Janeiro. The sample comprised 37 patients over 18 years old, who had been on hemodialysis or peritoneal dialysis at the hospital for three months or more. Data were collected using a patient satisfaction questionnaire. Results: overall patient satisfaction was 3.89 for the three items: education, trust, and theoretical and practical skill, with hemodialysis patients returning 3.69 overall satisfaction, and peritoneal dialysis patients, 4.44 . Conclusion: patients in peritoneal dialysis at the hospital were satisfied with the nursing care provided.

Descriptors: Patient satisfaction; nursing; nephrology; nursing care.

\section{RESUMEN}

Objetivo: analizar la satisfacción de los pacientes en tratamiento de diálisis en relación con los cuidados de enfermería. Método: estudio descriptivo, de abordaje cuantitativa de campo, realizado entre mayo y agosto de 2016, en un hospital universitario de Río de Janeiro. La muestra consistió en 37 pacientes en hemodiálisis o diálisis peritoneal en el hospital por tiempo igual o superior a tres meses, mayores de 18 años. Se utiliza, para la recolección de datos, el instrumento de satisfacción del paciente. Resultados: el promedio de satisfacción general de los pacientes fue de 3,89 para los tres ítems: educación, confianza y teórico-práctico. Los pacientes de hemodiálisis presentaron un promedio de satisfacción de 3,69 y los pacientes de diálisis peritoneal de 4,44. Conclusión: los pacientes en tratamiento de diálisis del hospital están satisfechos con la atención de enfermería que se les proporciona.

Descriptores: Satisfacción del paciente; enfermería; nefrología; atención de enfermería.

\section{INTRODUÇÃo}

A doença renal crônica (DRC) é uma anormalidade da estrutura ou função renal que consiste em uma síndrome clínica presente por um período igual ou superior a três meses, sendo adotadas para seu estadiamento a taxa de filtração glomerular e a albuminúria ${ }^{1}$.

Para seu enfrentamento, é necessário que haja a intervenção da equipe de saúde a fim de diminuir a morbidade e mortalidade causadas pela DRC, permitindo a otimização do diagnóstico precoce da doença, encaminhamento imediato para tratamento nefrológico e implementação de medidas para preservar a função renal auxiliem nos cuidados com o paciente atingido ${ }^{2}$.

O enfermeiro ao ser inserido na especialidade da nefrologia tem ampliado seu campo de atuação onde

'Enfermeira. Residente de Enfermagem, Universidade do Estado do Rio de Janeiro. Brasil. E-mail: ingridfoliveira@hotmail.com. "Enfermeiro. Professor Assistente, Universidade do Estado do Rio de Janeiro. Brasil. E-mail: felipe.santos@uerj.br.

"'Enfermeira. Doutora em Ciências da Saúde. Professora Adjunta, Universidade do Estado do Rio de Janeiro. Brasil. E-mail: francesvcs@gmail.com. IVEnfermeira. Doutora em Enfermagem. Professora Assistente, Universidade do Estado do Rio de Janeiro. E-mail: silviamarialins@gmail.com.

vEnfermeira. Residente de Enfermagem, Universidade do Estado do Rio de Janeiro. Brasil. E-mail: natasha.cunha84@yahoo.com 
exerce a assistência, o gerenciamento, a responsabilidade legal, social, de ensino, pesquisa, a promoção, contribuindo para a recuperação da saúde do paciente e sua capacidade de enfrentamento em cada tipo de tratamento ${ }^{3,4}$. Possui também um grande comprometimento diante da qualidade do cuidado que presta ao paciente, à instituição, fundamentado na ética, nas leis, nas normas da profissão, na valorização do cuidado e satisfação dos pacientes ${ }^{5}$.

O cuidar do enfermeiro envolve o agir, pensar e relacionar sua assistência focada no ser humano, identificando as necessidades individuais de cada paciente e proporcionando meios de atendimento que se adequarão ao tratamento deste indivíduo, contribuindo dessa maneira uma melhor qualidade de vida do mesmo. O enfermeiro, ao prestar sua assistência, reflete suas representações e individualidades nas diferentes relações, como na terapêutica, na confiança e na técnica, estabelecendo assim um relacionamento interpessoal, que influenciará na adesão ao tratamento e prolongamento da vida desse sujeito ${ }^{6,7}$.

Uma estratégia utilizada pelas organizações de saúde para apreender sobre a satisfação do paciente é o Instrumento de Satisfação dos Pacientes (ISP), que consiste em avaliar a percepção do paciente, sua expectativa e satisfação em relação à qualidade do cuidado de enfermagem e a qualidade do serviço de saúde prestado sob os aspectos sociais do cuidado, o caráter intrapessoal e o aspecto operacional do enfermeiro, que visa obter informações referentes à qualidade da atenção recebida pelo paciente ${ }^{5,8}$.

O estudo teve como principal objetivo analisar a satisfação dos pacientes em tratamento dialítico com relação aos cuidados de enfermagem. Como objetivos específicos, este trabalho visa apresentar o grau de satisfação do paciente em tratamento dialítico com os cuidados de enfermagem e discutir as diferenças e semelhanças das dimensões educação, confiança e conhecimento teórico-prático dos pacientes submetidos à hemodiálise (HD) e à diálise peritoneal (DP).

Seu potencial de contribuição para a área está baseado na associação do ensino, da pesquisa e da prática ao buscar destacar possíveis facilitadores e dificultadores da relação enfermeiro- paciente. Ao conhecer a satisfação do paciente, o enfermeiro pode estabelecer uma maior compreensão sobre sua clientela, favorecendo a construção de um elo de confiança com o sujeito e consequentemente uma melhoria, readaptação ou continuidade do cuidado que é prestado, contribuindo para a obtenção de avaliação da instituição e da própria assistência de enfermagem.

\section{REVISÃO DE LITERATURA}

Quando se relaciona o cuidado e a assistência do enfermeiro na área da nefrologia, a atuação dos profissionais se destaca em algumas áreas específicas desta especialidade: na atuação com pacientes ainda em tratamento conservador, na atuação com pacientes que irão ser, serão ou são submetidos ao transplante renal e nas duas modalidades de tratamento dialítico: hemodiálise e diálise peritoneal.

Ao cuidar de pessoas com DRC, o enfermeiro deve atentar para as complicações da doença, estresse e ansiedade que envolvem essa condição. Inicialmente, quando o indivíduo ainda encontra-se no tratamento conservador, os objetivos se basearão no auxílio da redução do ritmo de progressão da doença renal, na manutenção da função renal e na melhoraria das condições clínicas, psicológicas e sociais do sujeito ${ }^{9}$.

O enfermeiro no contexto do transplante renal busca orientar e auxiliar o paciente na adesão ao tratamento e acompanhamento, encontrar o melhor meio de passar as orientações, buscando a participação conjunta do paciente e sua família; buscar a melhor maneira de lutar contra os problemas que podem ser inevitáveis; exercer o papel assistencial e de educação de forma a contribuir de maneira significativa ao êxito do transplante e sempre que possível, acompanhar o paciente desde o início da evolução de sua enfermidade até alcançar uma melhor qualidade de vida através do procedimento e perceber as possíveis deficiências encontradas, estando junto ao paciente e atuando estrategicamente ${ }^{10}$.

A DP é a modalidade de terapia renal substitutiva (TRS) que mais se destaca quando se fala do autocuidado. O paciente deve assumir o controle de seu próprio tratamento e ter consciência do seu próprio cuidado através de atividades desenvolvidas por ele para realizar os procedimentos da diálise, os cuidados com o cateter e seu entorno, prevenção das complicações, dentre outros. Neste contexto, o enfermeiro assume um importante papel educativo: preparar pacientes e familiares para assumir os procedimentos da diálise, encorajando-os a serem responsáveis pelo seu cuidado, com a ajuda da família e dar autonomia a este cuidado ${ }^{11}$.

$\mathrm{Na} \mathrm{HD}$, o enfermeiro ao preparar o indivíduo para o procedimento, deve estabelecer uma relação de confiança com o mesmo e buscar seu encorajamento para questionar, aprender a evitar complicações, tirar dúvidas e promover o autocuidado, que contribuirá de maneira específica na integridade, no equilíbrio das funções orgânicas e na preservação do acesso vascular. O profissional deve incentivar o paciente a não ser passivo no tratamento, impulsionando-o a atentar para as alterações físicas e fisiológicas que ocorrem durante a evolução da $\mathrm{DRC}^{12}$.

Ao ouvir o que os pacientes têm para relatar sobre o cuidado que lhes é prestado e sobre sua satisfação, a chance de construção de um indicador de resultado que aponte aos gestores alguns caminhos decisórios de transformações e inovações se evidencia. O enfermeiro, por sua vez, pode adquirir consciência da sua responsabilidade diante da qualidade do cuidado que presta ao paciente, à instituição, à ética, às leis e às normas da profissão, bem como contribui na valorização e desempenho do cuidado e satisfação dos pacientes ${ }^{5}$. 


\section{Metodologia}

Trata-se de um estudo descritivo e transversal de abordagem quantitativa, realizado em uma instituição universitária localizada no município do Rio de Janeiro, Estado do Rio de Janeiro, onde funciona a especialidade de nefrologia subdividido em três setores: HD, DP e enfermaria de nefrologia clínica e de transplante renal.

A amostra foi constituída de 37 sujeitos, sendo utilizados como critério de inclusão os portadores de DRC que realizam os tratamentos dialíticos (DP e HD) vinculados à instituição com tempo de tratamento igual ou maior que três meses, de ambos os sexos e maiores de 18 anos. Foram excluídos da pesquisa pacientes em HD com doença renal aguda, pacientes transplantados que não necessitam mais de HD e/ou DP e pacientes que não conseguiram responder o questionário.

A coleta dos dados foi realizada no período de maio a agosto de 2016, no próprio setor de nefrologia. Os participantes da pesquisa foram os pacientes que compareceram ao centro para consultas de DP ou para a realização da HD.

Após a explicação dos objetivos da pesquisa e, em caso positivo de participação, a assinatura do Termo de Consentimento Livre e Esclarecido (TCLE) foi solicitada e o questionário a ser respondido foi entregue.

Caso o paciente possuísse alguma deficiência (visual, por exemplo) ou alguma dificuldade de compreensão do questionário, que interferisse em sua autoaplicação, era solicitada a ajuda da pesquisadora.

O projeto foi aprovado pelo Comitê de Ética e Pesquisa do Hospital Universitário Pedro Ernesto/UERJ, sob parecer no 1.436.717. Os aspectos da Resolução no 466/2012 do Conselho Nacional de Saúde foram respeitados.

Para a coleta de dados, foi utilizado como questionário o Instrumento de Satisfação do Paciente (ISP). Trata-se de um instrumento adaptado e validado no Brasil, a partir do original desenvolvido por Hinshaw e Atwood (1982) para ser aplicado em pacientes internados, sendo denominado de Patient Satisfaction Instrument (PSI). Possui como objetivo mensurar a satisfação do paciente com os cuidados de enfermagem ${ }^{8}$.

É composto por 25 questões fechadas que buscam avaliar a satisfação do paciente em relação aos domínios: área técnica-profissional, relacionamento educacional e confiança; os itens são respondidos mediante escores da escala de cinco pontos do tipo Likert, variando de concordo totalmente (cinco pontos) para discordo totalmente (um ponto), sendo que, quanto maior a pontuação, maior o nível de satisfação ${ }^{13}$.

Para o domínio técnico-profissional e confiança, a pesquisa conta com sete itens para cada, em que aborda questões técnicas do cuidado e situações relacionadas às atitudes do enfermeiro, respectivamente. Para o domínio confiança, a pesquisa traz 11 itens sobre situações envolvendo o relacionamento interpessoal entre o enfermeiro e o paciente ${ }^{13}$.

As respostas aos questionários foram digitadas, constituindo um banco de dados no programa Exce/ $2007^{\circledR}$. Para a análise estatística descritiva, utilizou-se o programa STATA MP 13.0, que forneceu a média aritmética e o intervalo de confiança dos resultados das variáveis obtidas.

\section{RESUltados E Discussão}

Os resultados apresentados apontam que a maioria dos entrevistados era do sexo masculino, com 20 (54,05\%) sujeitos. A faixa etária predominante estava entre os 51 a 60 anos, com $8(21,62 \%)$ pacientes, seguido da faixa etária de 61 a 70 anos, com 7 (18,92\%). A maioria dos entrevistados possuia Ensino Médio completo, com 12 (33.33\%) pessoas, seguido de Ensino Fundamental incompleto, com 9 (25\%), conforme mostra a Tabela 1.

TABELA 1: Caracterização dos sujeitos entrevistados. Rio de Janeiro, 2016. (37)

\begin{tabular}{|c|c|c|}
\hline Variáveis & $\mathbf{n}$ & $\%$ \\
\hline \multicolumn{3}{|l|}{ Sexo } \\
\hline Masculino & 20 & 54.05 \\
\hline Feminino & 17 & 45.95 \\
\hline \multicolumn{3}{|l|}{ Faixa etária } \\
\hline De 11 a 20 anos & 3 & 8.11 \\
\hline De 21 a 30 anos & 4 & 10.81 \\
\hline De 31 a 40 anos & 2 & 5.41 \\
\hline De 41 a 50 anos & 6 & 16.22 \\
\hline De 51 a 60 anos & 8 & 21.62 \\
\hline De 61 a 70 anos & 7 & 18.92 \\
\hline De 71 a 80 anos & 5 & 13.51 \\
\hline Mais de 80 anos & 2 & 5.41 \\
\hline \multicolumn{3}{|l|}{ Escolaridade $(n=36)$} \\
\hline Analfabeto & - & - \\
\hline Fundamental incompleto & 9 & 25 \\
\hline Fundamental completo & 4 & 11.11 \\
\hline Médio incompleto & 7 & 19.44 \\
\hline Médio completo & 12 & 33.33 \\
\hline Superior & 4 & 11.11 \\
\hline \multicolumn{3}{|l|}{$\mathrm{TRS}^{(*)}$ atual } \\
\hline Hemodiálise & 27 & 72.97 \\
\hline Diálise Peritoneal & 10 & 27.03 \\
\hline \multicolumn{3}{|l|}{ Tempo na atual TRS $(n=35)$} \\
\hline De 3 a 6 meses & 9 & 25.71 \\
\hline De 6 meses a 1 ano & 2 & 5.71 \\
\hline De 1 a 2 anos & 5 & 14.29 \\
\hline De 2 a 3 anos & 4 & 11.43 \\
\hline De 3 a 5 anos & 8 & 22.86 \\
\hline 5 anos ou mais & 7 & 20 \\
\hline \multicolumn{3}{|c|}{ Já realizou outra modalidade de TRS? } \\
\hline Sim & 13 & 35.14 \\
\hline Não & 24 & 64.86 \\
\hline \multicolumn{3}{|c|}{$\begin{array}{l}\text { Se sim, quanto tempo permaneceu } \\
\text { no outro TRS? }(n=13)\end{array}$} \\
\hline Menos de 3 meses & 2 & 15.38 \\
\hline De 3 a 6 meses & 2 & 15.38 \\
\hline De 6 meses a 1 ano & 2 & 15.38 \\
\hline De 1 a 2 anos & 2 & 15.38 \\
\hline De 2 a 3 anos & - & - \\
\hline De 3 a 5 anos & - & - \\
\hline 5 anos ou mais & 5 & 38.46 \\
\hline
\end{tabular}

${ }^{(*}$ TRS = Terapia renal substitutiva 
O maior número de pacientes entrevistados realizava HD, com 27 (72,97\%). Já os pacientes que realizam a terapia de DP foram 10 (27,03\%).

A média aritmética e os intervalos de confiança dos valores encontrados sobre a satisfação dos pacientes entrevistados em relação aos cuidados de enfermagem nas terapias renais substitutivas estão relacionados na Tabela 2 .

Os pacientes, no geral, encontravam-se satisfeitos nos três aspectos analisados: confiança, educação, teórico-prático, com a média de 3,89 de uma escala que varia de 1 a 5, sendo que, quanto maior a pontuação, maior o nível de satisfação dos entrevistados.

Os dados reforçam a importância do papel do enfermeiro durante o processo de cuidar. Ao construir um vínculo com o paciente, o profissional estabelece com este uma relação de confiança e a segurança perante o cuidado recebido. Isso favorece a comunicação e a consequente melhoria na qualidade da assistência que é prestada. Vale salientar que a avaliação do paciente referente ao cuidado prestado é baseada em situações que envolvem a atenção, confiança, empatia e envolvimento por parte do profissional que presta sua assistência e não somente na realização dos procedimentos técnicos e que a avaliação se dá de maneiras diferentes pois depende da singularidade de cada indivíduo ${ }^{8}$.

Observa-se que o aspecto que contou com menor pontuação foi relacionado à educação. $O$ indivíduo com DRC necessita ser orientado sobre diversos aspectos de seu tratamento: a enfermidade em si, as formas de terapia renal substitutiva, riscos e benefícios de cada modalidade de terapia, dieta, uso de medicamentos, restrição hídrica, dentre outros. A orientação fornecida através de ações educativas é essencial para viabilizar o autocuidado, aumentar a adesão ao esquema terapêutico, diminuir as intercorrências decorrentes do tratamento e auxiliar a identificação das necessidades do cliente, que interferem na sua qualidade de vida ${ }^{14}$.

TABELA 2: Satisfação geral dos pacientes em relação aos cuidados de enfermagem nas terapias renais substitutivas. Rio de Janeiro, 2016.

\begin{tabular}{lccc}
\hline $\begin{array}{c}\text { Satisfação em relação } \\
\text { à }\end{array}$ & $\mathbf{n}$ & $\begin{array}{c}\text { Média } \\
\text { aritmética }\end{array}$ & $\begin{array}{c}\text { Intervalo de } \\
\text { confiança }\end{array}$ \\
\hline Confiança (C) & 37 & 3.90 & $3.70-4.10$ \\
Educação (E) & 37 & 3.77 & $3.54-4.00$ \\
Teórico-prático (TP) & 37 & 4.01 & $3.80-4.22$ \\
Geral (C+E+TP) & 37 & 3.89 & $3.69-4.09$ \\
\hline
\end{tabular}

O enfermeiro possui como responsabilidade participar ativamente da implantação de programas educacionais que atendam às necessidades individuais dos pacientes, acompanhar sua trajetória, evolução e refletir sobre seus comportamentos para auxiliá-los a usufruir da qualidade de vida possível dentro de seu quadro e estado de saúde ${ }^{14}$.

A satisfação dos pacientes, em cada uma das modalidades de terapia renal substitutiva, hemodiálise e diálise peritoneal, é demonstrada na Tabela 3.

Percebe-se que, apesar da não equivalência quantitativa de pacientes em cada modalidade, os pacientes da DP possuem maior média de satisfação geral $(4,44)$ que os pacientes da HD $(3,69)$. Em cada um dos três aspectos, se analisados separadamente, os pacientes da DP também apresentaram maiores médias que os pacientes da HD.

Um dos fatores que pode justificar a diferença dos valores encontrados é a dinâmica própria da DP, cujo atendimento está ancorado numa relação próxima entre enfermeiro e paciente, desde o processo de capacitação para a diálise em domicilio, estreitando vínculos de apoio e responsabilização. Uma outra leitura está no fato dos pacientes que realizam a DP terem contato duas vezes por mês, aproximadamente, com os profissionais da clínica. Já os pacientes da HD realizam a terapia no local três vezes por semana, com duração média de quatro horas, o que possibilita maiores situações de estresse entre paciente e funcionários ${ }^{15}$.

A sistematização das intervenções autônomas do enfermeiro nas grandes áreas da especialidade de nefrologia se realizam quando o profissional passa a integrar uma abordagem holística na representação de uma perspectiva global dos cuidados. Esta permite incluir outras áreas de atenção direcionadas para a pessoa nas suas dimensões, com o objetivo de proporcionar a capacitação da mesma para o autocuidado, a identificação de complicações e a realização do tratamento no contexto da nefrologia ${ }^{16}$.

No contexto da DP, o enfermeiro considera como foco de seu cuidado a realização da capacitação específica do paciente e seu cuidador para o procedimento e o autocuidado, além do acompanhamento rotineiro dessa prática, através das consultas de enfermagem. Ao informar, incentivar e apoiar no processo de adaptação às exigências do método, o profissional proporciona o alcance de um bom nível geral de informação a respeito da DP e suas peculiaridades, o paciente é levado à compreensão de que método é um universo dotado de inúmeras especificidades ${ }^{17}$.

TABELA 3: Satisfação dos pacientes em cada modalidade de terapias renais substitutivas em relação aos cuidados do enfermeiro. Rio de Janeiro, 2016.

\begin{tabular}{|c|c|c|c|c|c|c|}
\hline \multirow{2}{*}{ Satisfação em relação à } & \multicolumn{3}{|c|}{ Hemodiálise } & \multicolumn{3}{|c|}{ Diálise peritoneal } \\
\hline & $\mathbf{n}$ & Média & $I C^{(*)}$ & $\mathbf{n}$ & Média & IC \\
\hline Confiança & 27 & 3.69 & $3.49-3.90$ & 10 & 4.46 & $4.17-4.74$ \\
\hline Educação & 27 & 3.57 & $3.31-3.82$ & 10 & 4.31 & $3.96-4.66$ \\
\hline Teórico-prático & 27 & 3.80 & $3.58-4.03$ & 10 & 4.57 & $4.31-4.82$ \\
\hline Geral (C+E+TP) & 27 & 3.69 & $3.47-3.90$ & 10 & 4.44 & $4.17-4.72$ \\
\hline
\end{tabular}

${ }^{(*)} I C=$ Intervalo de confiança. 
O enfermeiro ajudará na existência de um fator de proteção, o que culminará a uma melhor qualidade de aplicação da técnica dialítica. Ao existir uma relação entre paciente-profissional, é possível aprofundar, de forma única e específica, as expectativas, desejos e sentimentos do paciente, que culminará no aprofundamento de questões pertinentes à DP, seus cuidados e peculiaridades ${ }^{17,18}$.

$\mathrm{Na} \mathrm{HD}$, o foco do cuidado se dá principalmente durante a diálise, onde o paciente, o dialisador e o dialisato precisam de constante monitoração e avaliação. O enfermeiro desempenha a importante função de vigilância, para evitar complicações que podem ocorrer durante o procedimento, como embolia gasosa, ultrafiltração inadequada ou excessiva (hipotensão, câimbra, vômitos), extravasamentos sanguíneos e contaminações no acesso, o que demanda constante atenção e tempo do profissional ${ }^{12}$.

Um grande desafio para o enfermeiro atuante na HD é o preparo do paciente, pois este pode não compreender o impacto da diálise e as necessidades de aprendizado podem passar despercebidas, o que dificulta o autocuidado, que estabelece ações que seguem um modelo de recomendações, contribuindo de maneira específica na integridade, no equilíbrio das funções orgânicas e na preservação do acesso vascular ${ }^{12}$. O enfermeiro, além de atuar junto aos pacientes na implementação desse procedimento, desempenha um importante papel na educação desse indivíduo ao orientá-lo sobre o funcionamento da HD, o que facilitará as ações para a busca do autocuidado, na implementação da terapêutica medicamentosa e nutricional, ajudando-o a compreender a importância de adesão ao tratamento para que o paciente possa viver com bem-estar ${ }^{14}$.

Por fim, ressalta-se que os profissionais devem se preocupar em estudar a qualidade do seu cuidado, pois é através da percepção dos pacientes que se realiza uma troca entre a prática do cuidado, o que permite conhecer os aspectos considerados mais significativos ao ser assistido. Ao levar em conta que a percepção do cuidado do enfermeiro varia em função das características dos pacientes, da cultura, expectativas, fatores pessoais e da enfermidade, faz-se necessário que os enfermeiros meçam estas influências para realizar ajustes e seguir com a assistência oferecida, de maneira a examinar os pontos baixos e superá-los para, assim, garantir a qualidade assistencial e a satisfação dos clientes ${ }^{19-21}$.

\section{CONCLUSÃo}

Conclui-se que os pacientes em tratamento dialítico do hospital se encontram satisfeitos com os cuidados do enfermeiro que lhes são prestados.

Entretanto faz-se necessário que os profissionais atuantes na área conheçam os resultados obtidos e seus significados, para que possam refletir, revê-los ou dar prosseguimento ao seu cuidado, assegurando a sua qualidade.

Os aspectos confiança, educação e conhecimento teórico-prático, analisados pela pesquisa, são itens que fazem parte constante da assistência do enfermeiro. Vale ressaltar que o item que atingiu o menor índice de satisfação, seja no índice geral ou no índice de cada uma das duas modalidades dialíticas foi o de educação, cabendo a indagação: as estratégias utilizadas são suficientes para se realizar a educação em saúde com os pacientes? Há recursos suficientes (pessoal, material, financeiro) para se praticar a educação em saúde com esses pacientes? Há um real interesse por parte do profissional de realizar a educação em saúde?

O estudo teve como limitações a não equivalência quantitativa de sujeitos entrevistados em cada modalidade dialítica, e a reduzida amostra, a qual não permite a generalização dos achados.

Por fim, reconhecer os resultados apresentados no estudo contribuem para que a qualidade do serviço dos enfermeiros atuantes nas unidades de HD e DP da instituição de saúde seja reconhecida, mesmo diante das dificuldades enfrentadas pelo hospital e pelo serviço.

\section{REFERÊNCIAS}

1. Magalhães FG, Goulart RMM. Doença renal crônica e tratamento em idosos: uma revisão integrativa. Rev. Bras. Geriatr. Gerontol. 2015; 18: 679-92.

2. Bastos MG, Kirsztajn GM. Doença renal crônica: importância do diagnóstico precoce, encaminhamento imediato e abordagem interdisciplinar estruturada para melhora do desfecho em pacientes ainda não submetidos à diálise. J. Bras. Nefrol. 2011; 33: 93-108. 3. Pennafort VPS, Furtado AM, Fialho AVM, Moreira TMM, Freitas MC, Queiroz MVO. Produção do conhecimento científico de Enfermagem em Nefrologia. Rev. bras. enferm. (Online). 2010; 63: 830-36.

4. Carvalho IMP, Melo RL, Andraus LMS. Produção científica de enfermagem em nefrologia, no Brasil, no período de 1989 até 1999. Rev. eletron. enferm. 2001 [Acesso em 18 set. 2017]; 3. Disponível em: http://www.fen.ufg.br/revista.

5. Freitas JS, Silva AEBC, Minamisava R, Bezerra ALQ, Sousa MRG. Qualidade dos cuidados de enfermagem e satisfação do paciente atendido em um hospital de ensino. Rev. latinoam enferm. (Online). 2014; 22: 528-30.

6. Rodrigues TA, Botti NCL. Cuidar e o ser cuidado na hemodiálise. Acta Paul. Enferm. (Online). 2009; 22: 528-30.

7. Bisca MM, Marques IR. Perfil de diagnósticos de enfermagem antes de iniciar o tratamento hemodialítico. Rev. bras. enferm. 2010; 63: 435-9.

8. Oliveira AML, Guirardello EB. Satisfação do paciente com os cuidados de enfermagem: comparação entre dois hospitais. Rev. Esc. Enferm. USP. 2006; 40: 71-7.

9. Roso CC, Beuter M, Kruse MHL, Girardon-Perlini NMO, Jacobi CS, Cordeiro FR. O cuidado de si de pessoas em tratamento conservador da insuficiência renal crônica. Texto \& contexto enferm. 2013; 22: 739-45.

10. Furtado AMO, Souza SROS, Oliveira BL, Garcia CN. El enfermero asistencial y educador en una unidad de trasplante renal: un desafío. Enfermería Global. 2012; 27: 346-50.

11.Sadala MLA, Bruzos GAS, Pereira ER, Bucuvic EM. A experiência vivida pelos pacientes em DP domiciliar: uma abordagem fenomenológica. Rev. latino-am enferm. 2012; 20: 68-75.

12. Moreira AGM, Araújo STC, Torchi TS. Preservação da fístula arteriovenosa: ações conjuntas entre enfermagem e cliente. Esc. Anna Nery Rev. Enferm. 2013; 17: 256-62.

13. Oliveira AML. Satisfação do paciente com os cuidados de enfermagem: adaptação cultural e validação do Patient Satisfaction 
Instrument [dissertação de mestrado]. Campinas (SP): Universidade Estadual de Campinas; 2004.

14. Santos I, Rocha RPF, Berardinelli LMM. Necessidades de orientação de enfermagem para o autocuidado de clientes em terapia de hemodiálise. Rev. bras. enferm. 2011; 64: 335-42.

15. Gonçalves FA, Dalosso IF, Borba JMC, Bucaneve J, Valerio NMP, Okamoto CT, et al. Qualidade de vida de pacientes renais crônicos em hemodiálise ou diálise peritoneal: estudo comparativo em um serviço de referência de Curitiba - PR. J. Bras. Nefrol. 2015 ; 37: 467-74. 16. Sousa CN. Cuidar da pessoa com fístula arteriovenosa: modelo para a melhoria contínua. Revista Portuguesa de Saúde Pública. 2012; 30: 11-7.

17. Santos FK, Valadares GV. Conhecendo as estratégias de ação e interação utilizadas pelos clientes para o enfrentamento da DP. Esc. Anna Nery. Rev. Enferm. 2013; 17: 423-31.

18. Santos FK, Valadares GV. Conhecendo o mundo do ser que enfrenta a diálise peritoneal: nexos simbólicos presentes no cotidiano. Rev. enferm. UERJ. 2011; 19:473-8.

19. Contreras CCT. Percepción de la calidad del cuidado de enfermería en pacientes hospitalizados. Avances en enfermería. 2010; 28: 98-110.

20. Abreu IS, Santos CB. Qualidade de vida relacionada à saúde de pacientes em hemodiálise. Rev. enferm. UERJ. 2013; 21(1):95-100 21.Sousa MRG, Silva AEBC, Bezerra ALQ, Freitas JS, Neves GE, Paranaguá TTB. Prevalência de eventos adversos em uma unidade de hemodiálise. Rev. enferm. UERJ. 2016; 24(6):e18237. 